Abstracta Iranica

Revue bibliographique pour le domaine irano-aryen

Volume 37-38-39 | 2018

Comptes rendus des publications de 2014-2016

\title{
Muriel Debié. L'écriture de l'histoire en syriaque. Transmissions interculturelles et constructions identitaires entre hellénisme et islam
}

Christelle Jullien

\author{
(2) OpenEdition \\ Journals \\ Édition électronique \\ URL : http://journals.openedition.org/abstractairanica/42890 \\ DOI : $10.4000 /$ abstractairanica. 42890 \\ ISBN : 1961-960X \\ ISSN : 1961-960X \\ Éditeur : \\ CNRS (UMR 7528 Mondes iraniens et indiens), Éditions de l'IFRI
}

\section{Référence électronique}

Christelle Jullien, « Muriel Debié. L'écriture de I'histoire en syriaque. Transmissions interculturelles et

constructions identitaires entre hellénisme et islam », Abstracta Iranica [En ligne], Volume 37-38-39 | 2018, document 2, mis en ligne le 30 décembre 2018, consulté le 28 septembre 2020. URL : http://

journals.openedition.org/abstractairanica/42890; DOI : https://doi.org/10.4000/abstractairanica. 42890

Ce document a été généré automatiquement le 28 septembre 2020.

Tous droits réservés 


\title{
Muriel Debié. L'écriture de l'histoire en syriaque. Transmissions interculturelles et constructions identitaires entre hellénisme et islam
}

\author{
Christelle Jullien
}

\section{RÉFÉRENCE}

Muriel Debié. L'écriture de l'histoire en syriaque. Transmissions interculturelles et constructions identitaires entre hellénisme et islam. (Late Antique History and Religion 12), Louvain : Peeters, 2015, 724 p. ISBN 978-90-429-3237-1.

1 Cet ouvrage est à la fois un outil qui facilitera la recherche sur la littérature historique rédigée en syriaque, et un livre de réflexion sur l'écriture de l'histoire en milieu syriaque. Il fournit un important répertoire descriptif de textes et d'auteurs, tout en abordant des questions méthodologiques sur les enjeux de l'écriture historique jusqu'à la Renaissance syriaque aux $\mathrm{XII}^{\mathrm{e}}$ et $\mathrm{XIII}^{\mathrm{e}}$ siècles. L'historiographie syriaque a été produite par des auteurs issus de communautés professant des christologies diversifiées (syro-orthodoxes, chalcédoniens, maronites ou syro-orientaux), et ces choix doctrinaux ont souvent conditionné leur manière de comprendre et d'écrire l'histoire.

Deux parties structurent le volume. La première est une synthèse structurée en douze chapitres thématiques consacrés à la présentation des acteurs de l'historiographie en syriaque et au contexte matériel de leurs productions, les supports, les influences, les transmissions culturelles et les modes de diffusion de ces textes, en situant l'écriture historique au centre du processus de formation de l'identité syriaque. Peut-être eut-il été intéressant aussi d'aborder la question des encres (fabrication, couleurs, usages) et des éléments décoratifs. À une identification des auteurs et des textes (bien que 
beaucoup soient anonymes) et une caractérisation des genres historiques, l'A. ajoute une étude sur quelques lieux d'écriture en milieu monastique mais aussi scholastique urbain: en Syrie et en Osrhoène avec Amid, Mélitène et Édesse, et quelques "métropoles d'Iran et d'Iraq", Nisibe, Karkā d-Bēth Slokh (la moderne Kirkouk), Arbèles (Erbil). On aurait pu ajouter l'école de Séleucie, Dorqonie, Gundešābuhr, BēthQoqa, Rabban-Šābuhr... pour compléter cette esquisse cartographique. Le chapitre 4 propose une sélection d'auteurs de livres historiques (néanmoins tous miaphysites) avec présentation biographique (Jean d'Éphèse, Théophile d'Édesse, Denys de TellMahrrē, Michel le Syrien, Bar 'Ebrōyō). Dans les chapitres suivants (6-7), il est question de la mesure du temps, des systèmes chronologiques utilisés par les historiens syriaques et de leur conception du temps : détermination des usages calendaires et des cycles de Pâque, référence aux différentes ères, décompte du temps biblique. Une attention est portée aux sources bibliques, grecques et islamiques des histoires syriaques. Outre les textes narratifs, hagiographiques et ce que l'A. appelle les documents (archives, correspondances, informations tirées de témoignages oraux et d'observations, inscriptions), l'historiographie grecque est particulièrement étudiée en raison de l'influence majeure de son approche sur la production syriaque (p. 288-340), spécialement à travers l'œuvre ecclésiastique d'Eusèbe de Césarée. L'A. distingue la rédaction de textes relevant du genre proprement historique d'autres récits sur le passé, écrits par exemple sous la forme de biographies, en la rattachant aux traditions historiographiques grecques et aux sources eusébiennes. Celles-ci concernent aussi bien les histoires séculières qu'ecclésiastiques, ainsi que les chroniques universelles ou locales. Cette approche "eusébienne" dont héritèrent les historiens syriaques, qui associe aux événements de l'histoire de l'Église les grandes figures chrétiennes, marque le point focal de la réflexion de l'A. sur la question. Si l'on peut regretter qu'elle soit un peu disséminée dans plusieurs chapitres, elle peut être considérée aussi comme le "fil rouge" de la démonstration. D'autres modélisations dans les formes de discours sur le passé auraient pu être davantage mises en évidence, spécialement les types historiques ou bibliques sous-jacents aux hagiographies, aux martyrologies et aux vies de saints, et l'on soulignera d'une manière générale la faible part dévolue dans le volume au genre hagiographique, pourtant porteur d'une importante mémoire historique. Celui-ci est un peu abordé du point de vue des récits syro-orientaux (p. 354-356) mais surtout syrooccidentaux - les p. 356-358 et p. 404-408, qui relèvent de problématiques similaires (sur la construction des héros par exemple), auraient pu être regroupées pour faciliter la navigation dans l'ouvrage et la lecture. D'une manière générale, l'appareil des notes infra-marginales est extrêmement réduit dans cette première partie: davantage de références à la recherche scientifique nationale et internationale en cours sur laquelle s'appuie à l'évidence l'A. dans ses discussions thématiques auraient été appréciées. Le chapitre 10 est consacré aux transmissions interculturelles - c'est aussi le sous-titre de l'ouvrage. Essentiellement, il s'agit d'une étude exemplifiée de la transmission des données historiques d'abord au sein de la tradition syro-occidentale (p. 380-381), dans les chroniques byzantines où sont intégrés des matériaux syriaques (surtout une discussion sur la Chronographie de Théophane le Confesseur et la Chronique de Georges le Syncelle), dans les emprunts aux textes chrétiens par les auteurs de sources islamiques et inversément ; l'A. donne quelques exemples de ce processus, en particulier avec la Chronique de Théophile et ses insertions (p. 399-402). Peut-être aurait-il fallu regrouper ces éléments dans la notice consacrée à cet auteur (p. 139-143) où sont déjà évoqués les littérateurs qui se sont servis de son œuvre et le contexte de sa rédaction? On se serait 
aussi attendu à une meilleure visibilité des études de S. Brock sur les transmissions du grec vers le syriaque et vice-versa. Par ailleurs, l'évocation des transmissions de textes historiographiques syriaques vers d'autres corpus littéraires orientaux (caucasien, copte ou éthiopien), dont on sait les liens très forts avec la tradition syriaque, n'a pas été suggérée. Voir sur le sujet l'ouvrage édité par F. Jullien, Eastern Christianity: a Crossroads of Cultures, (Eastern Christian Studies 16), Louvain: Peeters, 2012; A. Vacca, "The Fires of Naxčawan. In Search of Intercultural Transmission in Arabic, Armenian, Greek, and Syriac », Le Muséon 129/3-4, 2016, p. 323-362.

3 La seconde partie est formée de plusieurs annexes dont un répertoire de presque $150 \mathrm{p}$. recensant les différentes chroniques ou ouvrages historiques - certains, perdus, n'étant connus que par de simples mentions dans d'autres œuvres (p. 505-647). Quatre rubriques détaillent les textes grecs traduits en syriaque, les textes syro-occidentaux (la plus développée), syro-orientaux et syro-arabes. Chaque œuvre fait l'objet d'une fiche technique comportant des indications sur son titre en syriaque, son auteur, une bibliographie sélective, et les principales éditions et traductions ; d'autres données sont souvent ajoutées : date de rédaction, forme littéraire, manuscrits où se trouve le texte, période concernée. Précisons que la plupart des éditions et traductions des textes historiographiques conservés sont disponibles en ligne sur le site internet syri.ac sous l'onglet " chronicles and historiography " (http://www.syri.ac/chronicles). Deux cartes des lieux de production historiographique en syriaque ( $\mathrm{VI}^{\mathrm{e}}$-IX ${ }^{\mathrm{e}}$ et $\mathrm{X}^{\mathrm{e}}$-XIV $\mathrm{V}^{\mathrm{e}}$ siècles) sont également fournies, avec une synopse chronologique des textes. La bibliographie est divisée en trois listes, qui n'incluent pas les références propres à chaque œuvre (placées s.v.) : les sources historiques non syriaques citées dans le corps de l'ouvrage, les catalogues de manuscrits syriaques, et la littérature moderne dont les références ont été abrégées dans les notes. Cette dispersion des informations bibliographiques à travers tout l'ouvrage nuit beaucoup à la clarté des renvois et complexifie considérablement les recherches... lorsqu'elles n'aboutissent pas parfois à des apories : la référence à l'article de Grenet "Les Sassanides à Doura Europos » (n. 119 p. 26) est introuvable ; de même pour van Rompay «A Letter of the Jews » (n. 95 p. 118), pour les Actes de Mar Mari (n. 122 p. 26), pour Horn, Asceticism and Christological Controversy (n. 4 p. 405), etc. Certaines références sont citées via une collection et non un auteur ( CSCO $»$ n. 81 p. 172: en l'occurrence ici Guidi, éditeur de la Chronique d'Édesse). Ajoutons certaines petites incohérences de classement dans la bibliographie (van Roey à « $\mathrm{V}$ » / van Rompay à « $\mathrm{R}$ »; de Halleux à « $\mathrm{H}$ » / di Branco à « $\mathrm{D}$ »). On s'étonnera de ne pas voir cités les deux ouvrages de la collection Études syriaques: L'historiographie syriaque, (Études syriaques 6), Paris: Geuthner, 2009, édité par l'A. où se trouve les premiers éléments d'une bibliographie des sources historiques syriaques p. 211-217; et Le monachisme syriaque, éd. F. Jullien, qui fournit également une bibliographie détaillée p. 305-332. Ajoutons aussi, certes ancien mais important pour l'état de l'art, S. P. Brock, "Syriac Historical Writing: A Survey of the Main Sources ", Journal of the Iraq Academy (Syriac Corporation) 5, 1979, p. 1-30. Un index des manuscrits cités, et un index général complètent l'ensemble. Cette vaste synthèse est une contribution importante à notre compréhension du développement de l'historiographie en milieu syriaque entre héritage grec et milieu arabe, et son répertoire forme un instrument désormais indispensable. 


\section{AUTEURS}

\section{CHRISTELLE JULLIEN}

CNRS, Mondes iranien et indien, Paris 